ORIGINAL ARTICLE

\title{
Histopathological assessment of lymph nodes in colorectal carcinoma: does triple levelling detect significantly more metastases?
}

\author{
C Verrill, N J Carr, E Wilkinson-Smith, E H Seel
}

J Clin Pathol 2004;57:1165-1167. doi: 10.1136/icp.2004.018002

See end of article for authors' affiliations .....................

Correspondence to: Dr C Verrill, Department of Histopathology, Southampton General Hospital, Southampton University Hospitals NHS Trust, Tremona Road, Southampton SO16 6YD, UK; clareverrill@hotmail. com

Accepted for publication 20 April 2004
Aims: Standard practice is to take one section from every lymph node found in colorectal carcinoma resection specimens, to look for metastatic carcinoma. This study evaluates whether assessing three sections separated by $100 \mu \mathrm{m}$ detects significantly more metastases in nodes than the conventional single section.

Methods: A retrospective study of 100 colorectal carcinoma resection specimens. All blocks containing lymph nodes had two extra histological sections cut (separated by $100 \mu \mathrm{m}$ ) and stained with haematoxylin and eosin. The original slide was called level 1, and the extra two sections levels 2 and 3.

Results: Twenty Dukes's A (equivalent to WHO-UICC stage grouping I, pTNM stage pT1/2N0), 43 Dukes's B (equivalent to WHO-UICC stage grouping II, pTNM stage pT3/4N0), and 37 Dukes's C (equivalent to WHO-UICC stage grouping III, pTNM stage at least pN1) cases were examined (total 1453 nodes). Twelve extra metastases (in 11 patients) were discovered in nodes at levels 2 and 3, which were negative in level 1. Ten cases were Dukes's $C$ and, in one patient, this led to upstaging from N1 to N2 (pTNM classification system). One case was Dukes's B and the discovery of a single metastasis on level 2 upstaged it to Dukes's C.

Conclusions: Triple levelling detected more tumour deposits than the conventional single section. In two patients, the staging classification of the lesion was changed, with potentially important implications for prognosis and management.
A pproximately 35000 people develop colorectal carcinoma in the UK every year, making it the third most common cancer in the UK. ${ }^{1}$ Patients usually undergo surgical resection for their disease and resection specimens are submitted to a histopathology department for staging. Two staging systems are commonly used; Dukes's classification and the pTNM classification system of tumours. ${ }^{23}$ Accurate staging is essential because it provides the basis for further treatment, such as chemotherapy, and provides prognostic information for patients. Metastasis to regional lymph nodes is one of the most important factors relating to the prognosis of colorectal carcinoma. ${ }^{4}$ Patients with tumour deposits in regional lymph nodes are staged as Dukes's $\mathrm{C}$ and as $\mathrm{Nl}$ or $\mathrm{N} 2$ in the pTNM classification system $(\mathrm{Nl}$ is metastasis in one to three regional lymph nodes, N2 is metastasis in four or more regional lymph nodes).

\section{"Metastasis to regional lymph nodes is one of the most important factors relating to the prognosis of colorectal carcinoma"}

There are currently no guidelines in the Royal College of Pathologists' minimum data set ${ }^{5}$ for colorectal carcinoma on whether one haematoxylin and eosin (H\&E) section is sufficient for the assessment of lymph node metastases. The Association of Directors of Anatomic and Surgical Pathology in the USA has recommended that one H\&E section for each cassette containing lymph nodes should be cut. ${ }^{6}$ We are unaware of convincing evidence for the assessment of more than one histological section when looking for lymph node metastases in colorectal carcinoma. The situation is different in the subspecialty area of breast pathology, however, where there are specific evidence based guidelines issued by The National Health Service Breast Screening Programme on the assessment of lymph nodes retrieved from axillary tissue in the setting of primary breast cancer. ${ }^{7}$ These guidelines recommend that lymph nodes smaller than $5 \mathrm{~mm}$ should be embedded in entirety and examined at two levels, and lymph nodes larger than this should be cut into three slices and one node processed for each cassette. This is based on publications from groups such as the international (Ludwig) breast cancer study group, which found that $9 \%$ of axillary lymph nodes judged to be negative on one routine histological section were subsequently found to contain metastases if they were subjected to levelling. ${ }^{8}$ Usual practice in colorectal specimens is for several small lymph nodes to be placed into each cassette and then one H\&E section to be cut from it for microscopic assessment. In our histopathology laboratory in Southampton, UK, a standard set of levels would comprise three levels cut at $100 \mu \mathrm{m}$ intervals.

We hypothesised that examining three histological sections separated by $100 \mu \mathrm{m}$ would detect significantly more tumour metastases in lymph nodes from colorectal carcinoma resection specimens than the standard practice of examining one section.

\section{MATERIALS AND METHODS}

One hundred consecutive patients who underwent surgical resection for adenocarcinoma of the colon or rectum received by the histopathology department at Southampton General Hospital, UK, from l January 2003 were identified. The original slides and paraffin wax blocks were retrieved from the archives and unlinked and anonymised. An extra two levels (separated by $100 \mu \mathrm{m}$ ) were cut from any paraffin wax

Abbreviations: $H \& E$, haematoxylin and eosin 
blocks containing lymph nodes. The original H\&E section (from the archives) was then counted as the first level and the extra two sections cut for this study became the second and third levels. All the sections were then examined for the presence of tumour metastases within lymph nodes at all three levels. The presence of any extra metastases detected in the second and third levels that were not present in the first level was noted. These were counted as metastases that would have remained undetected if only the first level had been examined. We determined whether the discovery of extra metastases in the second and third levels led to the upstaging of the specimen in the Dukes's or pTNM classification systems. Any tumour cells visible on H\&E stained sections were counted as a metastasis; no attempt was made to distinguish between metastases and micrometastases. The maximum diameter of each lymph node was also recorded.

\section{RESULTS}

One hundred cases of adenocarcinoma of the colon and rectum were examined: 20 Dukes's A (equivalent to WHOUICC stage grouping I, pTNM stage pTl/2N0), 43 Dukes's B (equivalent to WHO-UICC stage grouping II, pTNM stage pT3/4N0), and 37 Dukes's C (equivalent to WHO-UICC stage grouping III, pTNM stage at least pN1). One thousand four hundred and fifty three lymph nodes were examined and the mean average number of lymph nodes for each patient was 14.5 (range, 1-43). Twenty three per cent of lymph nodes were greater than $5 \mathrm{~mm}$ in diameter. Three hundred and forty seven paraffin wax blocks containing lymph nodes (mean average, 3.5/patient) were cut, resulting in an extra 694 sections. The mean average number of lymph nodes for each paraffin wax block was 4.2 (range, l-21). Eleven of the 100 patients had extra tumour metastases detected on levels 2 and 3 that were undetected on level 1 (one of these patients had two extra metastases). This included 10 cases of Dukes's $\mathrm{C}$, of which nine had no change to their staging as a result of this finding, but one was upstaged from $\mathrm{Nl}$ to $\mathrm{N} 2$ in the pTNM tumour classification system. One case of Dukes's B had a single tumour metastasis detected on level 2 (as a small subcapsular sinus deposit), which was not present in level 1 , and this led to the upstaging of this case from Dukes's B to Dukes's C and from No to $\mathrm{Nl}$ in the pTNM system. The current pTNM recommendation is for 12 lymph nodes to be assessed for each patient. In this study, 53 of the 100 patients had more than 12 lymph nodes assessed. In particular, in the two patients who were upstaged, nine lymph nodes were assessed for each.

Of the 12 extra metastases detected, eight were found on level 2 and a further four were found on level 3 only. Eight of these were deposits within the parenchyma of the lymph node and four were subcapsular sinus deposits (fig 1). Three of these extra metastases occurred in newly appearing lymph nodes that had not been present in the first level (lymph node diameters 1,3 , and $3 \mathrm{~mm}$ ) and nine occurred in lymph nodes that were present in level 1 , but contained no visible tumour deposits. The sizes of the 12 newly discovered tumour deposits were $0.1,0.2,0.25,0.4,0.6,0.6,0.8,1.4,1.5,2.0,3.4$, and $4.5 \mathrm{~mm}$ in maximum diameter.

\section{DISCUSSION}

In our study, 11 of the 100 patients examined had extra tumour metastases discovered in levels 2 and 3 of the lymph nodes that would have gone undetected if only 1 level had been examined, as is the current practice. Ten of the 11 cases were Dukes's C in level 1 and only in one patient did the detection of extra metastases in levels 2 and 3 result in upstaging from $\mathrm{N} 1$ to $\mathrm{N} 2$ (pTNM). None of the extra metastases detected were in apical lymph nodes. In these patients, the detection of extra metastases is probably academic, with little bearing on prognosis or further treatment. However, the situation is different for the patient with Dukes's B colorectal carcinoma, in whom we discovered the only lymph node metastasis in level 2, which upstaged the tumour to Dukes's C and therefore would have important implications for management and prognosis.

Our study is consistent with the findings of previous studies investigating the use of triple levels on lymph nodes in colorectal carcinoma. Van Wyk et al examined 72 cases of colorectal carcinoma prospectively (with the lymph nodes being sectioned at three levels with an interval of $100 \mu \mathrm{m}$ ) and discovered four cases containing extra tumour metastases in levels 2 and 3. ${ }^{9}$ Three of these were Dukes's C and this did not change the staging. In one case, a single lymph node metastasis was discovered on levels 2 and 3, which upstaged the tumour from Dukes's B to Dukes's C. This study differs from ours in that they followed the protocol for dealing with axillary lymph nodes set out by the National Health Service Breast Screening Programme, which makes the findings less applicable to everyday practice, because this protocol is not followed for colorectal carcinoma resections specimens, and practice varies between pathologists as to how lymph nodes are placed into cassettes. Therefore, we cut levels on any block containing lymph nodes, however they had been placed into cassettes, and whatever the size. Our study did not require the processing of extra tissue blocks.

\section{"Our study shows the benefit of performing three levels on lymph nodes in colorectal carcinoma resection specimens separated by $100 \mu \mathrm{m}$ to obtain an accurate picture of the number of lymph nodes containing tumour metastases"}

Other publications include a study of triple levelling on 33 cases of Dukes's A and B carcinoma, which detected no extra metastases,$^{10}$ and a study of 11 cases of colon carcinoma,
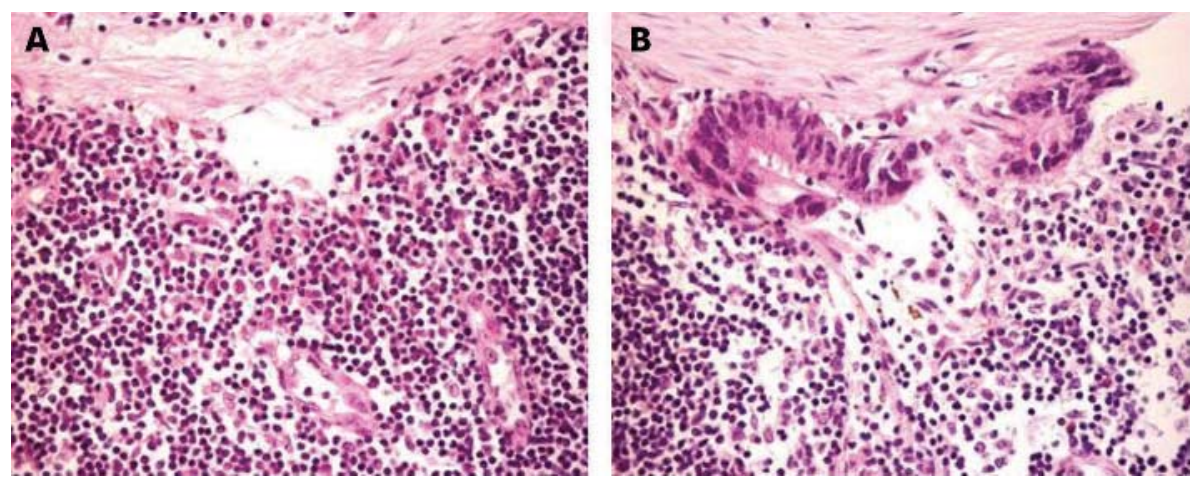

Figure 1 A lymph node (stained with haematoxylin and eosin) from a patient with Dukes's C colorectal carcinoma. (A) At level 1, there are no visible tumour deposits (this was also the case in level 2). (B) At level 3, there is a small subcapsular sinus deposit of metastatic tumour visible. 


\section{Take home messages}

- When assessing lymph nodes found in colorectal carcinoma resection specimens, cutting three sections separated by $100 \mu \mathrm{m}$ detected more tumour deposits than the conventional single section

- In two of 100 patients, the staging classification of the lesion was changed, with potentially important implications for prognosis and management

- We believe that the small increase in workload required for triple levelling is justified by the increased accuracy in staging

which detected one further positive case when a more detailed examination with three additional sections was undertaken. ${ }^{11}$ Hitchcock et al describe a study using extra H\&E sections plus cytokeratin immunocytochemistry to detect colorectal cancer metastases, ${ }^{12}$ but their methods were different from those used in our study.

Pathology laboratories are under constant workload pressure as a result of staff shortages and financial constraints, and therefore it is important that histopathologists use evidence based practice before ordering extra studies, such as deeper levels that increase this workload. We believe that our study shows the benefit of performing three levels on lymph nodes in colorectal carcinoma resection specimens separated by $100 \mu \mathrm{m}$ to obtain an accurate picture of the number of lymph nodes containing tumour metastases. The small increase in workload is justified by the increased accuracy in staging.

\section{Authors' affiliations}

C Verrill, N J Carr, E Wilkinson-Smith, E H Seel, Department of Histopathology, Southampton General Hospital, Southampton University Hospitals NHS Trust, Tremona Road, Southampton SO16 6YD, UK

\section{REFERENCES}

1 www.cancerresearchuk.org/aboutcancer/statistics/statstables/ colorectalcancer.

2 Dukes CE, Bussey $\mathrm{H}$. The spread of rectal cancer and its effects on prognosis. Br J Cancer 1958; 12:309-20.

3 The colon and rectum. In: Sobin LH, Wittekind CH, eds. TNM classification of malignant tumours, 6th ed. New York: Wiley-Liss, 2002:72-6.

4 Hobday TJ, Erlichman C. Colorectal cancer. In: Gospodarowicz MK, Henson DE, Hulter RVP, et al. Prognostic factors in cancer, 2nd ed. New York: Wiley Liss, 2001:267-79.

5 Quirke P, Williams GT. Standards and minimum datasets for reporting common cancers: colorectal cancer. The Royal College of Pathologists, July 1998.

6 Lawrence WD. ADASP recommendations for processing and reporting of lymph node specimens submitted for evaluation of metastatic disease. Virchows Arch 2001;439:601-3.

7 NHSBSP. Pathology reporting in breast cancer screening. NHSBSP Publication No 3 ed. Sheffield: NHSBSP Publications, 1995 (revised 1997).

8 International (Ludwig) Breast Cancer Study Group. Prognostic importance of occult axillary lymph node micrometastases from breast cancer. Lancet 1990:335:1565-8.

9 van Wyk Q, Hosie KB, Balsitis M. Histopathological detection of lymph node metastases from colorectal carcinoma. J Clin Pathol 2000;53:685-7.

10 Nicholson AG, Marks CG, Cook MG. Effect on lymph node status of triple levelling and immunohistochemistry with CAM5.2 on node negative colorectal carcinomas. Gut 1994:35:1447-8

11 Natsugoe S, Aiko T, Shimazu H. A detailed histological study on occult metastasis of the lymph nodes. Jpn J Surg 1991;21:528-32.

12 Hitchcock CL, Sampsel J, Young SX, et al. Limitations with light microscopy in the detection of colorectal cancer cells. Dis Colon Rectum 1999:42:1046-52. 